\title{
Entanglement and the lower bounds on the speed of quantum evolution
}

\author{
A. Borrás, ${ }^{1}$ M. Casas, ${ }^{1}$ A. R. Plastino, ${ }^{1,2,3}$ and A. Plastino ${ }^{2,3}$ \\ ${ }^{1}$ Departament de Física, Universitat de les Illes Balears and IMEDEA-CSIC, 07122 Palma de Mallorca, Spain \\ ${ }^{2}$ Department of Physics, University of Pretoria, 0002 Pretoria, South Africa \\ ${ }^{3}$ Observatory and Exact Sciences Faculty, National University La Plata, CONICET, C.C. 727, 1900 La Plata, Argentina
}

(Received 15 May 2006; published 28 August 2006)

The concept of quantum speed limit-time (QSL) was initially introduced as a lower bound to the time interval that a given initial state $\psi_{I}$ may need so as to evolve into a state orthogonal to itself. Recently [V. Giovanetti, S. Lloyd, and L. Maccone, Phys. Rev. A 67, 052109 (2003)] this bound has been generalized to the case where $\psi_{I}$ does not necessarily evolve into an orthogonal state, but into any other $\psi_{F}$. It was pointed out that, for certain classes of states, quantum entanglement enhances the evolution "speed" of composite quantum systems. In this work we provide an exhaustive and systematic QSL study for pure and mixed states belonging to the whole 15-dimensional space of two qubits, with $\psi_{F}$ a not necessarily orthogonal state to $\psi_{I}$. We display convincing evidence for a clear correlation between concurrence, on the one hand, and the speed of quantum evolution determined by the action of a rather general local Hamiltonian, on the other one.

DOI: 10.1103/PhysRevA.74.022326 PACS number(s): 03.67.Mn, 03.67.Lx, 03.65. $-\mathrm{w}$, 89.70.+c

\section{INTRODUCTION}

One of the most fundamental concepts in the quantum description of nature is that of entanglement [1-5], which in recent years has been the subject of intense research efforts (see, for instance, the following, by no means exhaustive list of references: [1-10]). A state of a composite quantum system is called "entangled" if it cannot be represented as a mixture of factorizable pure states. Otherwise, the state is called separable. Entanglement constitutes a physical resource that lies at the heart of important information processes [3-5] such as quantum teleportation, superdense coding, and quantum computation.

Entanglement is essential for both (i) our basic understanding of quantum mechanics and (ii) some of its most revolutionary (possible) technical applications. Thus, it is imperative to investigate in detail the relationships between entanglement and other aspects of quantum mechanics. In such a vein, particular interest is assigned to the exploration of the role played by entanglement with regards to the dynamical evolution of composite quantum systems.

In this effort we will be interested in the speed up of quantum evolution produced by entanglement. Why? Because in quantum computation one tries to (i) avoid loss of coherence and (ii) increase the velocity of information processing and information transmission. Anandan and Aharonov [11] have shown that, given a state $|\psi\rangle$ and a curve $C$ in the projective Hilbert space $\mathcal{P}$, the quantity

$$
s=2 \int d t \frac{\Delta E}{\hbar},
$$

with

$$
(\Delta E)^{2}=\left\langle\psi\left|H^{2}\right| \psi\right\rangle-\langle\psi|H| \psi\rangle^{2},
$$

is independent of the particular Hamiltonian $H$ used to transport the state along the curve and is in fact the distance along $C$ as measured by the Fubini-Study metric, deducing as a consequence the uncertainty relation

$$
(\Delta E) \Delta t \geqslant \frac{\hbar}{4}
$$

where $(\Delta E)$ is the time-averaged uncertainty in energy during the time interval $\Delta t$. Equality in Eq. (3) holds iff the system moves along a geodesic in $\mathcal{P}$. In this case the evolution may be said to have minimum uncertainty, analogous to how a Gaussian wave packet is said to have minimum position-momentum uncertainty at a given time. More generally they define an efficiency in evolution $\epsilon=s / s_{0}$, where the denominator gives the distance along the shortest geodesic joining the initial and final points of evolution. Loss of coherence in evolution may be regarded as due to the timeenergy uncertainty principle. In trying to avoid such loss, speeding up evolution seem advisable.

In this regard, Margolus and Levitin [12] have shown that the minimum evolution time in which one state evolves to an orthogonal one depends on the mean energy and the fluctuation. Giovannetti, Lloyd, and Maccone [1,2] recently uncover the fact that, in certain cases, entanglement helps to "speed up" the time evolution of composite systems. This "speed" of quantum evolution is also of considerable interest because of its relevance in connection with the physical limits imposed by the basic laws of quantum mechanics on the velocity of information processing and information transmission [12-15].

The evolution "speeding-up" ability of entanglement has been demonstrated only in special, if important, instances. One would like to ascertain that it is indeed an entanglement feature, and not just something that happens in these instances. Thus we will here undertake a general study. The aim of the present contribution is to make a systematic study of the connection between (a) entanglement and (b) the speed of quantum evolution as determined by the action of a rather general local Hamiltonian, by means of a numerical simulation. Our model belongs to a family that includes the basic models of quantum optics and cavity QED [16-19]. In a previous work [20] a corresponding study was performed just for (i) pure states of bipartite systems of low dimension- 
ality evolving towards (ii) an orthogonal state. Two different cases were analyzed: i) two qubits (distinguishable) systems and ii) bosonic or fermionic composite (bipartite) systems of the lowest dimensionality. In the present effort we are going to tackle an extension to the case of two distinguishable systems of (a) pure and (b) mixed states that evolve to (c) any other state, not necessarily orthogonal to the initial one. We also consider (d) the special case of maximum entangled mixed states (MEMS) [21], and also (e) that of the set of mixed states whose entanglement degree cannot be increased by the action of quantum gates (IH states) [22]. We remark on the facts that (i) MEMS have recently been detected experimentally $[23,24]$ and (ii) nowadays the possibility of obtaining such states via the action of local non-unitary quantum channels is being studied $[25,26]$. Thus, the ensuing results will be applicable to any physical systems where bipartite states play a leading role.

The paper is organized as follows: in Sec. II, using the time evolution of the fidelity we present the quantum speed limit for pure states. The case of mixed states is presented in Sec. III. The special case of MEMS and IH states are presented in Sec. IV, and finally some conclusions are drawn in Sec. V.

\section{QUANTUM SPEED LIMIT FOR PURE STATES}

Let us consider first the dynamical evolution of pure states for the case of two equal but distinguishable subsystems evolving under a local Hamiltonian, that is, we deal with a two-qubits system whose evolution is governed by the (local) Hamiltonian

$$
H=H_{A} \otimes I_{B}+I_{A} \otimes H_{B},
$$

whose eigenvalue equation writes

$$
\begin{gathered}
H_{A, B}=\epsilon_{A, B}|1\rangle, \\
H_{A, B}=0|0\rangle, \\
\epsilon_{A}=\delta_{A} \epsilon, \\
\epsilon_{B}=\delta_{B} \epsilon,
\end{gathered}
$$

$$
\epsilon \text { being an arbitrary energy, }
$$

Our bipartite states (the eigenstates of $H$ ) are $|00\rangle,|01\rangle$, $|10\rangle$, and $|11\rangle$, while the concomitant eigenvalues equal 0 , $\delta_{B} \epsilon, \delta_{A} \epsilon$, and $\left(\delta_{A}+\delta_{B}\right) \epsilon$, respectively.

\section{A. General methodological considerations}

In this paper we perform a systematic numerical survey of the evolution properties of arbitrary (pure and mixed) states of a two-qubits quantum system, under the action of the Hamiltonian (4), by recourse to an exhaustive exploration of the concomitant state-space $\mathcal{S}$. To such an end it is necessary to introduce an appropriate measure $\mu$ on this space. Such a measure is needed to compute volumes within $\mathcal{S}$, as well as to determine what is to be understood by a uniform distribu- tion of states on $\mathcal{S}$. The measure that we are going to adopt here is taken from the work of Zyczkowski et al. [27,28]. An arbitrary (pure or mixed) state $\rho$ of a quantum system described by an $N$-dimensional Hilbert space can always be expressed as the product of three matrices,

$$
\rho=U D\left[\left\{\lambda_{i}\right\}\right] U^{\dagger} .
$$

Here $U$ is an $N \times N$ unitary matrix and $D\left[\left\{\lambda_{i}\right\}\right]$ is an $N \times N$ diagonal matrix whose diagonal elements are $\left\{\lambda_{1}, \ldots, \lambda_{N}\right\}$, with $0 \leqslant \lambda_{i} \leqslant 1$, and $\Sigma_{i} \lambda_{i}=1$. The group of unitary matrices $U(N)$ is endowed with a unique, uniform measure: the Haar measure $\nu[29]$. On the other hand, the $N$-simplex $\Delta$, consisting of all the real $N$-uples $\left\{\lambda_{1}, \ldots, \lambda_{N}\right\}$ appearing in Eq. (6), is a subset of $a(N-1)$-dimensional hyperplane of $\mathcal{R}^{N}$. Consequently, the standard normalized Lebesgue measure $\mathcal{L}_{N-1}$ on $\mathcal{R}^{N-1}$ provides a measure for $\Delta$. The aforementioned measures on $U(N)$ and $\Delta$ lead then to a measure $\mu$ on the set $\mathcal{S}$ of all the states of our quantum system [27-29], namely,

$$
\mu=\nu \mathcal{L}_{N-1} .
$$

In our numerical computations we randomly generate pure and mixed states according to the measure (7).

\section{B. Pure states}

For pure states $|\Psi\rangle$ of our composite system the natural measure of entanglement is the usual reduced von Neumann entropy $S\left[\rho_{A, B}\right]=-\operatorname{Tr}_{A, B}\left(\rho_{A, B} \ln \rho_{A, B}\right.$ ) (of either particle $A$ or particle $B$ ) where $\rho_{A, B}=\operatorname{Tr}_{B, A}(|\Psi\rangle\langle\Psi|)$. It is convenient for our present purposes to use, instead of information measure $S\left[\rho_{A, B}\right]$ itself, the closely related concurrence value $C$, given by

$$
C^{2}=4 \operatorname{det} \rho_{A, B} .
$$

Both the entanglement entropy $S\left[\rho_{A, B}\right]$ and the concurrence $C$ are preserved under the time evolution determined by the local Hamiltonian (4). Given an initial state

$$
|\Psi(t=0)\rangle=c_{0}|00\rangle+c_{1}|01\rangle+c_{2}|10\rangle+c_{3}|11\rangle,
$$

with

$$
\sum_{i=1}^{4}\left|c_{i}\right|^{2}=1,
$$

its concurrence is,

$$
C^{2}=4\left|c_{0} c_{3}-c_{1} c_{2}\right|^{2} .
$$

Our objective is to characterize the departure of the system, at a time $t$ (represented by $\Psi(t)$ ), from its initial state $\Psi(t=0)$. To this end we can use the quantum concept of fidelity $P$ that, for pure states, is the squared-modulus of the overlap between the two states involved, i.e.,

$$
\begin{aligned}
P(z) & =|\langle\Psi(t=0) \mid \Psi(t)\rangle|^{2} \\
& =\left.|| c_{0}\right|^{2}+\left|c_{1}\right|^{2} z^{\delta_{B}}+\left|c_{2}\right|^{2} z^{\delta_{A}}+\left.\left|c_{3}\right|^{2} z^{\delta_{A}+a_{B}}\right|^{2},
\end{aligned}
$$

where

$$
z \equiv \exp (i \Omega),
$$

and 


$$
\Omega=\frac{t \epsilon}{\hbar}
$$

From now on we consider time intervals measured in units of $\hbar / \epsilon$, and use for this rescaled time the letter $\Omega$. We will also use the following notation: $\Delta^{+}=\delta_{A}+\delta_{B}$ and $\Delta^{-}$ $=\delta_{A}-\delta_{B}$

The key idea is that of measuring the speed of dynamical evolution by studying the time evolution of the fidelity. To such an end one first of all fixes a given $P$ amount, say $P$ $=F$, and proceeds to calculate the amount of time needed for a given state to evolve from $P(z)=1$ (at $t=0)$ to $P(z)=F$ at, say, $t=\tau$, for $F \in[0,1]$.

In Ref. [20] only the case $\delta_{A}=\delta_{B}=1$ was discussed. This particular Hamiltonian instance will be referred to as corresponding to the Hamiltonian $H_{I}$ in Sec. IV.

The condition (12) specializes for $H_{I}$ to

$$
\begin{aligned}
F= & 2 p_{03} \cos 2 \Omega+2 p_{03}\left(1-s_{03}\right) \cos \Omega \\
& +\left(1-s_{03}\right)^{2}+\left|c_{0}\right|^{4}+\left|c_{3}\right|^{4},
\end{aligned}
$$

where $p_{03}=\left|c_{0}\right|^{2}\left|c_{3}\right|^{2}$ and $s_{03}=\left|c_{0}\right|^{2}+\left|c_{3}\right|^{2}$.

In this case a minimum of the fidelity is achieved for the special value $\Omega=\Omega_{\text {min }}$ given by

$$
\Omega_{\text {min }}=\arccos \frac{-\left(1-s_{03}\right) s_{03}}{4 p_{03}},
$$

which can yield nonphysical complex values. To avoid this we limit the argument of the arccos to the interval $[-1,0]$.

The time $\tau$ required to evolve to a state with fidelity $F$ admits a lower bound that depends upon both the state's expectation energy $E$ and its fluctuation $\Delta E$ [2],

$$
T_{L . \text { Bound }}=\max \left(\alpha(F) \frac{\pi \hbar}{2 E}, \beta(F) \frac{\pi \hbar}{2 \Delta E}\right),
$$

where the functions $\alpha(F)$ and $\beta(F)$ are detailed in Ref. [2]. We can compute $\beta(F)$ using an expression previously proved in Refs. [30,31]

$$
\beta(F)=\frac{2}{\pi} \arccos (\sqrt{F}),
$$

while $\alpha(F)$ can be numerically calculated with great accuracy [2]. For $F=0$ (when the initial state evolves to an orthogonal one), one finds $\alpha(F)=\beta(F)=1$. In the opposite situation, when the state does not evolve $(F=1)$, both functions vanish.

In Ref. [20] situations were dealt with for which the orthogonal state to the initial one was definitely reached, which is not the usual case. A useful parametrization, introduced in this reference, reads

$$
\begin{gathered}
\left|c_{0}\right|^{2}=\left|c_{3}\right|^{2}=\Gamma, \\
\left|c_{1}\right|^{2}=-2 \delta \Gamma \cos \Omega \\
\left|c_{2}\right|^{2}=-2(1-\delta) \Gamma \cos \Omega,
\end{gathered}
$$

with $\Gamma=1 /[2(1-\cos \Omega)]$ and $\Omega \in\left[\frac{\pi}{2}, \pi\right]$, with $\delta \in[0,1]$. In other words, $\Omega=\arccos ((2 \Gamma-1) / 2 \Gamma)$. We note that introduc-
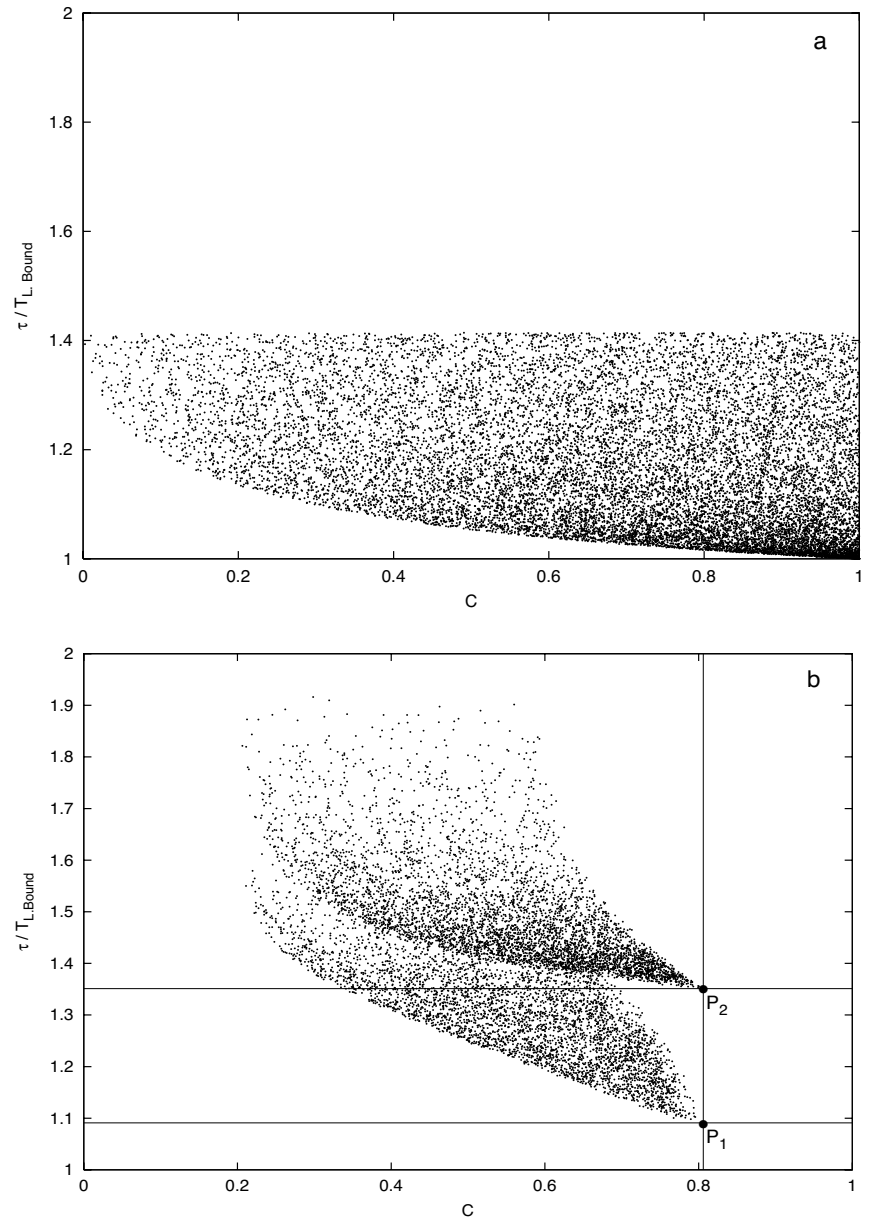

FIG. 1. $\tau / T_{L . \text { Bound }}$ for pure states. a) Pure states that evolve to an orthogonal one and b) pure states for which $F_{\min } \in[0.35,0.4]$. The points $P_{1}$ and $P_{2}$ represent the fastest states corresponding to each of the families determined by Eq. (17). For the range of values of $F_{\text {min }}$ considered here, these fast states correspond to $F_{\text {min }}=0.35$.

tion of this parametrization in the right-hand side of Eq. (16) for $F=0$ (orthogonality) does yield an equality.

To study the role of the entanglement on the speed of quantum evolution and on its lower bounds, one should pay attention to the $\left(C, \tau / T_{L . \text { Bound }}\right)$ plane. A representative group of two-qubit states evolving to an orthogonal one is depicted in Fig. 1(a). These states can easily be generated using the parametrization (19). The ratio $\tau / T_{L \text {.Bound }}$ has a maximum value equal to $\sqrt{2}$, no matter which value $C$ adopts. The minimum value of this ratio does strongly depend on $C$ through the value of $\Omega$, and can be analytically obtained [20]. Only maximally entangled states reach the bound $T_{\text {L.Bound }}$. Separable states have a different behavior. For a rather general Hamiltonian of type $H_{I}$, they all evolve to an orthogonal state in a fixed time $\tau / T_{L \text {.Bound }}=\sqrt{2}$.

These features can be easily explained. For pure states evolving to an orthogonal state according to $H_{I}$, the minimum time interval required to complete such evolution depends only on $\Delta E$. This is the first option in Eq. (17). In this specific case $\Delta E$ strictly depends on the value of $\Omega$ [20]. All separable states evolve to an orthogonal one in a fixed time $\Omega=\pi$, for which the minimum possible value of the ratio 
$\tau / T_{L . B o u n d}$ is $\sqrt{2}$. Thus, for separable states the maximum and the minimum of such ratio coincide. As the entanglement is increased, pure states are able to evolve in more rapid fashion. This is the reason that lies behind the dependence of the minimum of $\tau / T_{L \text {.Bound }}$ with the concurrence. Maximally entangled states can evolve to an orthogonal state in any time lapse within the range $\Omega \in\left(\frac{\pi}{2}, \pi\right)$. The states that evolve in the shortest possible time $(\Omega=\pi / 2)$ are also those to which a minimum of the ratio $\tau / T_{L . B o u n d}=1$ is assigned. Of course, we cannot extend the same conclusions to the rest of the states that evolve to some value of $F \neq 0$, as it is clearly seen in Fig. 1(b), or to other Hamiltonians than $H_{I}$.

From Eq. (15) we realize that the fidelity for pure states oscillates in time. Thus, as time goes on the fidelity of a given state climbs and goes down in alternating fashion, reaching minima of different depths. Our interest in this respect will be focused on those special times at which the first fidelity-minimum $F_{\min }$ is attained. Doing so we can treat all pure states in a unified manner. Note that the type of oscillation we are speaking about has a strong dependence on the form of the Hamiltonian one is dealing with.

We proceed to randomly generate initial states $|\Psi\rangle$ as explained above. For each state we calculate

(i) its concurrence $C_{\Psi}$ and

(ii) the first minimum $F_{\min }^{\Psi}$ that the fidelity attains during the time evolution of $|\Psi\rangle$. For a given pure state $|\Phi\rangle, F_{\text {min }}^{\Psi}$ tells us "how far" can $|\Phi\rangle$ travel in $\mathcal{S}$, before starting backwards towards itself, as guided by the Hamiltonian. Deeper valleys may be reached later in the periodic time evolution, but we are interested only in the one that is reached first.

(iii) the time $\tau$ required for the state $|\Psi\rangle$ to reach the first fidelity minimum $F_{\text {min }}^{\Psi}$,

(iv) the time interval $T_{L . \text { Bound }}$ that arises by the following process: fixing first an arbitrary $F$ value, $|\Psi\rangle$ can evolve to states $|\Phi\rangle$ such that the overlap (12) between $|\Psi\rangle$ and $|\Phi\rangle$ attains this value. Some time interval $t_{o}=\Omega \hbar / \epsilon$ [cf. Eq. (14)] is needed to reach each of these states. The minimum possible such interval is called $T_{L . B o u n d}^{\Psi}$ and given by the bound (17). Notice that the fidelity value $F$ reached in this time interval needs not correspond to any minimum of the fidelity.

Thus, for each $|\Psi\rangle$ we compute its concurrence $C_{\Psi}$, the (first) minimum of the fidelity $F_{m i n}^{\Psi}$, and the time $\tau$ (in units of $\left.T_{L . \text { Bound }}\right)$, i.e., the ratio $\tau / T_{L . \text { Bound }}$. This allows us to build up an association connecting each $|\Psi\rangle$ to these three quantities:

$$
|\Psi\rangle \rightarrow\left\{C, F_{\text {min }}, \tau / T_{L . B o u n d}\right\} .
$$

A representative group of those states for which $F_{\text {min }}$ $\in[0.35,0.4]$ is depicted in Fig. 1(b). Their behavior is quite different than those of Fig. 1(a). For these $F_{\min }$ values there exist two different families of states, corresponding to the two extant possibilities for $T_{L . B o u n d}$ [cf. Eq. (17)]. The lower one corresponds to those states for which the bound is determined by its expectation-energy value $E$ [the first one in Eq. (17)]. States for which the bound $T_{L . B o u n d}$ is determined by its energy spread $\Delta E$ belong to the upper group. None of these states (for both sets) reach the bound $\tau / T_{L . \text { Bound }}=1$. The maximum value for the ratio $\tau / T_{L . B o u n d}$ of Fig. 1(a) is clearly

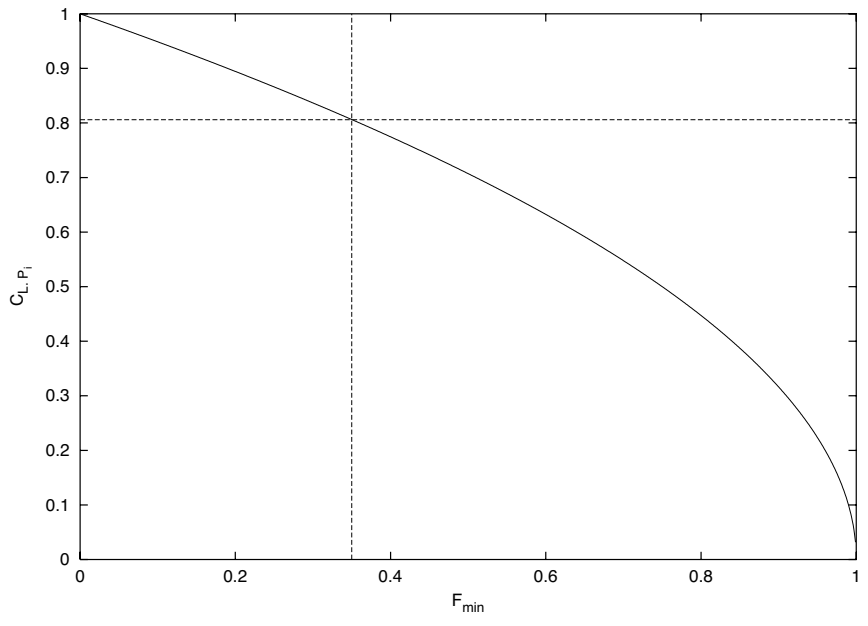

FIG. 2. Concurrence for the fastest pure states compatible with a given value of $F_{\min }$ as given by Eq. (22). The horizontal and vertical lines cross at the point corresponding to the fastest states (points $P_{1}$ and $P_{2}$ ) of Fig. 1(b).

exceeded here. There also exists a forbidden $C$ zone for states with $F_{\min }$ different than 0 . To acquire a global perspective regarding these families of states, for any value of $F_{\text {min }}$, we will study the dependence of their fastest evolving states $\left[P_{1}\right.$ and $P_{2}$ in Fig. 1(b)] with $F_{\min }$. These rapidly evolving states achieve the minimum of the fidelity in a time $\Omega_{\min }$ $=\pi / 2$. Also, from Eq. (15), we realize that the minimum of the fidelity cannot be reached in a time shorted than $\Omega_{\text {min }}$ $=\pi / 2$. If proper account of the normalization (10) of the initial state is taken, the only compatible state parametrization turns out to be

$$
\begin{gathered}
\left|c_{1}\right|^{2}=\left|c_{2}\right|^{2}=0, \\
\left|c_{0}\right|^{2}=\frac{1+\sqrt{F_{\text {min }}}}{2}, \\
\left|c_{3}\right|^{2}=\frac{1-\sqrt{F_{\text {min }}}}{2} .
\end{gathered}
$$

Given such a parametrization and minding Eq. (11), we ascertain that the concurrence $C_{L . P_{i}}$ for these fast states is the same for our two families, being completely determined once the value of $F_{\min }$ is fixed,

$$
C_{L . P_{i}}=\sqrt{1-F_{\text {min }}} .
$$

This dependence on $F_{\min }$ is illustrated if Fig. 2. For a given value of $F_{\text {min }}$, the concurrence $C_{L . P_{i}}$ of these fast states also coincides with the maximal concurrence value allowed for. Thus, determining the concurrence of the fastest states compatible with such fidelity is tantamount to finding the forbidden $C$ zone for such $F_{\min }$ value. Only in the special instance of states capable to reach (in their evolutiontrajectory) an orthogonal counterpart (i.e., $F_{\min }=0$ ) can we obtain any possible concurrence value. For the $F_{\min }$ range depicted in Fig. 1(b), the points $P_{1}$ and $P_{2}$ correspond to 


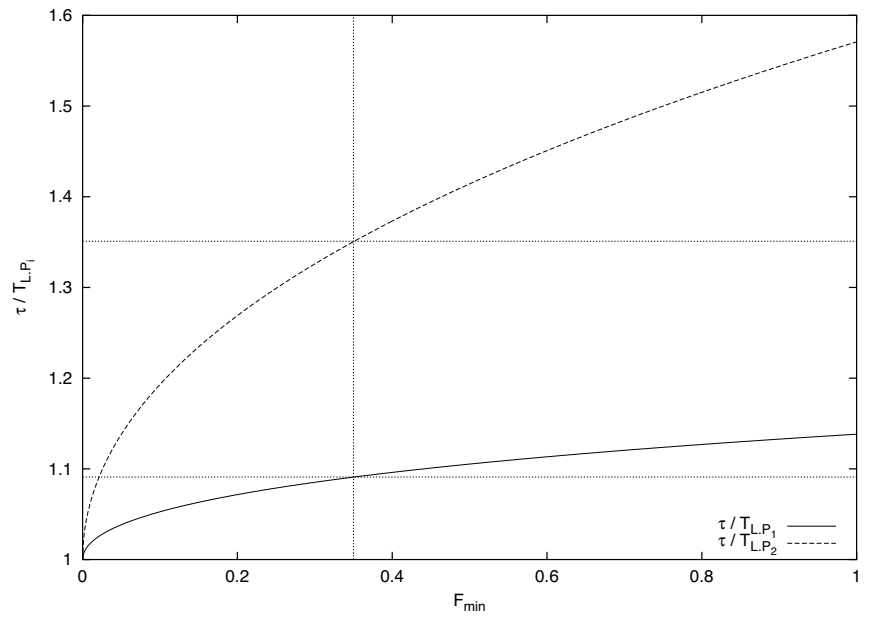

FIG. 3. $\tau / T_{L . P_{i}}$ for the fastest pure states compatible with a given value of $F_{\text {min }}$ as given by Eq. (24) for $\tau / T_{L . P_{1}}$ and by Eq. (25) for $\tau / T_{L . P_{2}}$. The horizontal and vertical lines cross at two different points corresponding to the fastest states (points $P_{1}$ and $P_{2}$ ) of Fig. 1(b). The upper crossing corresponds to $P_{2}$ and the lower one to $P_{1}$.

$F_{\text {min }}=0.35$. According to Eq. (22) their concurrence is $C_{L . P_{i}}$ $=0.806$, as shown in Figs. 1(b) and 2 .

Once we know the concurrence value for these special states, we ascertain the time (in $T_{\text {L.Bound }}$ units) required to reach $F_{\text {min }}$. We need first to compute the relation between $F_{\text {min }}$ and either the mean energy $E$ or the energy spread $\Delta E$. Using the parametrization (21) one obtains

$$
\begin{aligned}
& E=\epsilon\left(1-\sqrt{F_{\text {min }}}\right), \\
& \Delta E=\epsilon \sqrt{1-F_{\text {min }}} .
\end{aligned}
$$

Since we know that the shortest possible time needed to reach this $F_{\min }$ value is $\Omega_{\min }=\pi / 2$, using Eq (17) we easily find the ratio $\tau / T_{L . \text { Bound }}$ corresponding to the "fastest states" of our two families.

$$
\begin{aligned}
& \frac{\tau}{T_{L . P_{1}}}=\frac{1-\sqrt{F_{\text {min }}}}{\alpha\left(F_{\min }\right)} \\
& \frac{\tau}{T_{L . P_{2}}}=\frac{\sqrt{1-F_{\min }}}{\beta\left(F_{\min }\right)}
\end{aligned}
$$

Both quantities are depicted in Fig. 3. The higher the $F_{\text {min }}$ value, the more apart the two families get. They only coincide in the $F_{\text {min }}=0$ case, that is, for those states that evolve to an orthogonal one. We can apply these results to the special case considered in Fig. 1(b) $\left(F_{\min } \in[0.35,0.4]\right)$, remembering that in such $F_{\text {min }}$ range the points $P_{1}$ and $P_{2}$ correspond to $F_{\text {min }}=0.35$. For the fast state corresponding to point $P_{1}$ we have $E=0.408$ (in $\epsilon$ units) and $\tau / T_{L . P_{1}}=1.091$. For the state corresponding to the point $P_{2}$ one finds $\Delta E=0.806$ (in $\epsilon$ units) and $\tau / T_{L . P_{2}}=1.351$.

By recourse to numerical simulation we have also found that the number of states that evolve according to Eqs. (24) and (25) is a function of the value $F_{\min }$. For $F_{\min }=0$ the bound for all the involved states is given by $T_{L . B o u n d}$
$=\beta(F)(\pi \hbar / 2 \Delta E)$ [20]. For greater $F_{\text {min }}$ values the situation changes. If this value is large enough, approximately half of the states belong to one of the families, while the rest are accrued to the remaining one.

\section{QUANTUM SPEED LIMIT FOR MIXED STATES}

Given an initial mixed state $\rho(0)$ and using the Hamiltonian of the last section, we can easily calculate the corresponding density matrix of the system at a given time $t$

$$
\rho(t)=\left(\begin{array}{cccc}
\rho_{11} & \rho_{12} e^{i \delta_{B} \Omega} & \rho_{13} e^{i \delta_{A} \Omega} & \rho_{14} e^{i \Delta^{+} \Omega} \\
\rho_{21} e^{-i \delta_{B} \Omega} & \rho_{22} & \rho_{23} e^{-i \Delta^{-} \Omega} & \rho_{24} e^{i \delta_{A} \Omega} \\
\rho_{31} e^{-i \delta_{A} \Omega} & \rho_{32} e^{i \Delta^{-} \Omega} & \rho_{33} & \rho_{34} e^{i \delta_{B} \Omega} \\
\rho_{41} e^{-i \Delta^{+} \Omega} & \rho_{42} e^{-i \delta_{A} \Omega} & \rho_{43} e^{-i \delta_{B} \Omega} & \rho_{44}
\end{array}\right)
$$

where $\rho_{i j}=\rho_{i j}(0)$.

For mixed states the fidelity's expression adopts the well known expression

$$
F(\rho(0), \rho(t))=\{\operatorname{Tr}[\sqrt{\sqrt{\rho(0)} \rho(t) \sqrt{\rho(0)}}]\}^{2} .
$$

In the case of pure states, this fidelity reduces to the probability (12). For the case treated here, determined by the Hamiltonian $H_{I}$, the fidelity for pure states is given by Eq. (15) from which we realize that it oscillates in time. For mixed states such kind of analytical expression for the fidelity in not available, but one can compute the fidelity numerically and observe a similar behavior.

To study the case of mixed states we follow the same methodology used in the previous section for pure states. Thus, we randomly generate states $\rho$ in the two-qubits space of mixed states $\mathcal{S}$ (of 15 dimensions). We can thus classify the values of the ratio $\tau / T_{L . B o u n d}$ according to their corresponding values of the concurrence and the fidelity in such a mapping. We also fix our attention on the "concurrencefidelity" plane. Our numerical-sampling procedure will start by constructing a fine enough grid in the $(F, C)$ plane. We will have thus divided the plane into a large but discrete number of "windows." Each window, of course, contains many states $\rho$. We will assign to all of them the same pair of $(F, C)$ values. Notice that, for these distinct states $\rho, \tau$, and $T_{L . \text { Bound }}$ will in general be different. We thus average over them, but omit, for notational simplicity's sake, the " $<>$ " signs. The end-result is that we get a list of three quantities for each grid, namely,
1. $C$,
2. $F$,
3. $\tau / T_{\text {L.Bound }}$.

We also store information regarding the times $t$ at which the fidelity achieves some arbitrary fixed value, not necessarily connected with minima in any sense of the word. Specifically, for each $\rho$, we have selected intermediate values of the fidelity between its initial, and maximum, value $F=1$, and its (first-)minimum value $F_{\text {min }}^{\rho}$, according to intervals of size $0.05(F=1,0.95,0.9,0.85, \ldots)$. For these fidelities, we have stored the associated quantities $C, \tau / T_{L . \text { Bound }}$. 


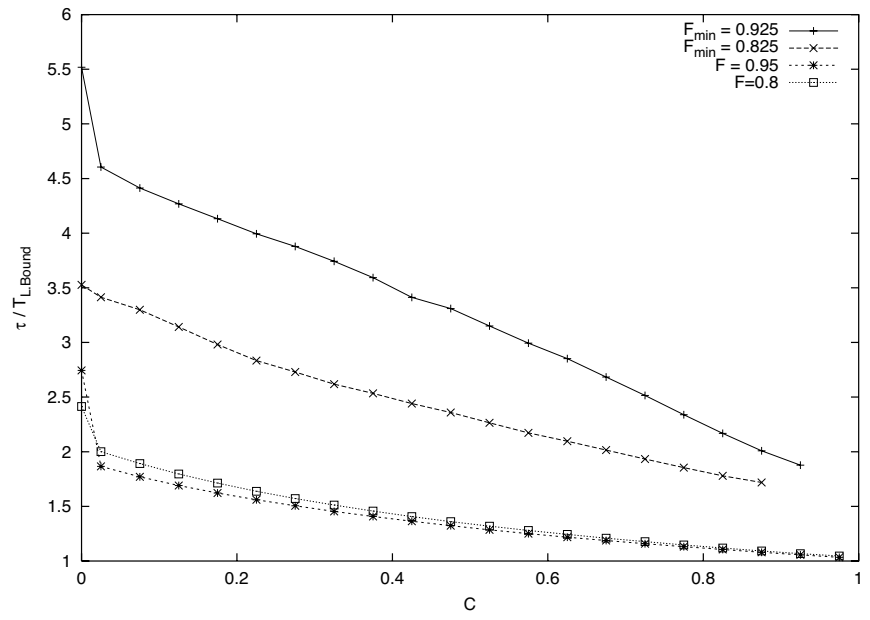

FIG. 4. $\tau / T_{L . B o u n d}$ for mixed states that evolve to several fixed $(F=0.95$ and $F=0.8)$ and minimum $\left(F_{\text {min }}=0.925\right.$ and $\left.F_{\text {min }}=0.825\right)$ values of the fidelity. See text for details.

As stated, the time evolution for mixed states is of a periodic nature and the oscillation strongly depends on the Hamiltonian form. One can circumvent to a considerable extent this $F$ dependence on the form by using only highfidelity values, for which, obviously, $F$ minima cannot be reached in arbitrarily small time intervals. A majority of the states $\rho \in \mathcal{S}$ attain these high-fidelity values but do not achieve, instead, lower ones. Using high-fidelity values is then tantamount to considering most of our randomly generated states $\in \mathcal{S}$.

Let us focus our attention upon $\tau / T_{L \text {.Bound }}$. As mentioned before, the quantity $T_{L . \text { Bound }}[\mathrm{cf}$. Eq. (17)] (also known as the quantum speed limit time) is the lower bound for the temporal interval required so as to evolve, from a state $\rho$ to a state $\sigma$, in such a manner that the pair $(\rho, \sigma)$ of companion states corresponds to a given fidelity $F$. We will first study this quantity for fixed fidelity values. In Fig. 4 we plot the value of $\tau / T_{\text {L.Bound }}$ vs the concurrence $C$ for mixed states evolving to high fidelity companion states. There exists a clear correlation between the quantum speed evolution time and the concurrence. The more entangled a state is, the less time it takes to reach a companion state such that the generalized overlap between them is $F$. Indeed, this time is seen to be close to the limit $\tau$, and $T_{L \text {.Bound }}$ for high $C$ values. The relation between $\tau / T_{L \text {.Bound }}$ and the concurrence $C$ does not seem to strongly depend on the specific $F_{\text {min }}$ value considered. Contrariwise, for pure states it is only when we consider small fidelity values that a clear correlation between $\tau / T_{L . \text { Bound }}$ and the concurrence $C$ is observed. These small fixed values of the fidelity are obviously very close to its corresponding $F_{\text {min }}$ value. Remind that we have shown in the previous section that the correlation between $\tau / T_{L \text {.Bound }}$ and the concurrence $C$ does exist for $F_{\text {min }}$. If one selects pure states with high fixed values of the fidelity $F$, the correlation we are here speaking about is not detected, as $\tau / T_{\text {L.Bound }}$ is approximately constant and close to unity for all possible values of the concurrence $C$.

Thus far we have considered arbitrary fidelities, not necessarily linked to minima of this quantity. In Fig. 4 we also consider time intervals $\tau$ needed to reach the first fidelity minimum. The resulting situation resembles the one just described above. The number of highly entangled $(C>0.9)$ mixed states evolving to companion states with a high value of the fidelity $(F>0.75)$ is very small. This entails that we cannot numerically obtain enough states in this zone to perform our averaging procedure in a reliable manner. Although the limit value $\tau / T_{L \text {.Bound }}=1$ is not reached for maximally entangled states, mixed states exhibit also in this case a nitid speed-concurrence correlation. Consequently, their evolution speed strongly depends on entanglement degree.

\section{MAXIMALLY ENTANGLED MIXED STATES (MEMS) AND IH STATES}

As we are interested in putative relations between entanglement and the speeding up of the quantum evolution, the study of some special types of states should be of interest. In particular, the so-called MEMS [21] are states that have the maximum possible amount of entanglement of formation. We remark on the fact that MEM states have recently been encountered in the laboratory $[23,24,26]$. The associated density matrix is written in terms of a variable $x$ that ranges in $[0,1]$. In the basis referred to in the Sec. II their representative matrices read

$$
\rho_{M E M S}=\left(\begin{array}{cccc}
g(x) & 0 & 0 & x / 2 \\
0 & 1-2 g(x) & 0 & 0 \\
0 & 0 & 0 & 0 \\
x / 2 & 0 & 0 & g(x)
\end{array}\right),
$$

with $g(x)=1 / 3$ for $0 \leqslant x \leqslant 2 / 3$, and $g(x)=x / 2$ for $2 / 3 \leqslant x$ $\leqslant 1$.

Also of great interest are the so-called Ishizaka and Hiroshima (IH) states [22], whose entanglement degree cannot be increased by acting on them with logic gates. Of course, MEMS are a special instance of the IH class. The associated $\rho_{I H}$ matrices, of eigenvalues $p_{i} ;(i=1,2,3,4)$, read

$$
\rho_{I H}=\left(\begin{array}{cccc}
p_{2} & 0 & 0 & 0 \\
0 & \frac{p_{3}+p_{1}}{2} & \frac{p_{3}-p_{1}}{2} & 0 \\
0 & \frac{p_{3}-p_{1}}{2} & \frac{p_{3}+p_{1}}{2} & 0 \\
0 & 0 & 0 & p_{4}
\end{array}\right),
$$

where the eigenvalues are size ordered: $p_{1} \geqslant p_{2} \geqslant p_{3} \geqslant p_{4}$. If one compares Eqs. (29) and (26) it is easy to see that IH states can "evolve" only if $\delta_{A} \neq \delta_{B}$. This entails that we cannot use here the same Hamiltonian $\left(H_{I}\right)$ employed in the preceding sections (see Sec. II). For IH states we will use the values $\delta_{B}=1$ and $\delta_{A}=2$, namely, we employ a local Hamiltonian $H_{I I}$, whose diagonal is $0, \epsilon, 2 \epsilon, 3 \epsilon$. For MEM states we will also use this Hamiltonian because we want to compare its associated results with those of the IH states. If one uses the Hamiltonian $H_{I}$ with MEM states the ensuing results resemble those of the preceding section. Thus, comparison 
can be made with the results of mixed states detailed there. In the MEMS instance the fidelity can readily be computed and reads

$$
F_{M E M S}=\left(A+\frac{1}{2}(\sqrt{B+\sqrt{C}}+\sqrt{B-\sqrt{C}})\right)^{2},
$$

with

$$
\begin{gathered}
A=1-2 g(x), \\
B=4 g(x)^{2}+x^{2} \cos \left(\Delta^{+} \alpha\right), \\
C=x^{2}\left(\cos \left(\Delta^{+} \alpha\right)-1\right)\left(8 g(x)^{2}+x^{2}\left(\cos \left(\Delta^{+} \alpha\right)-1\right)\right) .
\end{gathered}
$$

The MEMS's fidelity expression (31) also applies, with different coefficients, to the IH states case. Their corresponding coefficients are

$$
\begin{gathered}
A_{I H}=p_{2}+p_{4}, \\
B_{I H}=\left(p_{1}+p_{3}\right)^{2}+\left(p_{1}-p_{3}\right)^{2} \cos \left(\Delta^{-} \alpha\right), \\
C_{I H}=\left(1+\cos \left(\Delta^{-} \alpha\right)\right)\left(\left(p_{1}{ }^{2}+p_{3}{ }^{2}\right)\left(1+\cos \left(\Delta^{-} \alpha\right)\right)\right. \\
\left.+2 p_{1} p_{3}\left(3-\cos \left(\Delta^{-} \alpha\right)\right)\right) .
\end{gathered}
$$

The oscillating part of the MEMS fidelity seems to depend on $\cos \left(\delta_{A}+\delta_{B}\right) \Omega$ and the minimum fidelity value coincides with the minimum of its oscillating part, i.e., the minimum fidelity is achieved at $\Omega_{\min }^{M E M S}=\pi /\left(\delta_{A}+\delta_{B}\right)$. For the IH- states the situation is similar, the fidelity depends on $\cos \left(\delta_{A}-\delta_{B}\right) \Omega$ and its first minimum is achieved at $\Omega_{\text {min }}^{I H}$ $=\pi /\left(\delta_{A}-\delta_{B}\right)$.

We can also obtain an analytic expression for the expectation value of the hamiltonian $E$ and its fluctuation $\Delta E$ in the case of the MEMS states. We find

$$
\begin{gathered}
E_{M E M S}=\epsilon\left(\delta_{B}+g(x) \Delta^{-}\right), \\
\Delta E_{M E M S}=\epsilon \sqrt{g(x)\left(\delta_{B}{ }^{2}+\delta_{A}{ }^{2}\right)-g(x)^{2}\left(\Delta^{-}\right)^{2}} .
\end{gathered}
$$

For the Hamiltonian considered here $E$ is always greater than $\Delta E$. According to Eq. (17) (see also the paragraph following it) one looks for the maximum of a pair of quantities. Here $T_{L . B o u n d}$ is always equal to that one depending on $\Delta E$, because $\beta(F)$ is always greater than or equal to $\alpha(F)$. Taking into account all these results, the equation for $\tau$ turns out to read

$$
\frac{\tau}{T_{L . \text { Bound }}}=\frac{\Omega_{\min } \Delta E}{\epsilon \arccos \sqrt{F}}
$$

and one must substitute $\Delta E$ and $F$ with their pertinent associated values, depending on (i) which zone we are working in and (ii) which Hamiltonian we are referring to. For $\Omega_{\min }$ we obtain the values $\pi / 2$, for $\left(\delta_{B}=1, \delta_{A}=1\right)$, and $\pi / 3$, for $\left(\delta_{B}=2, \delta_{A}=1\right)$.

For the IH states we obtain the following equations for $E$ and $\Delta E$

$$
\begin{gathered}
E_{I H}=\frac{\epsilon}{2}\left(p_{1} \Delta^{-}+\left(p_{3}+2 p_{4}\right) \Delta^{+}\right), \\
\Delta E_{I H}=\frac{\epsilon}{2} \sqrt{2 p_{1} \Delta^{+} \Delta^{-}+2\left(p_{3}+2 p_{4}\right)\left(\Delta^{+}\right)^{2}-\left(p_{1} \Delta^{-}+\left(p_{3}+2 p_{4}\right) \Delta^{+}\right)^{2}} .
\end{gathered}
$$

Notice that the MEM states are completely determined by the parameter $x$, which corresponds to the value of the concurrence $(C=x)$. For a given value of the concurrence $C$ there exists only one value for that magnitudes we are interested on: $F_{\min }, E, \Delta E$, and $T_{L . B o u n d}$. This means that if we want to analyze these states we must do it using $F_{\min }$ instead of fixed, arbitrary values of the fidelity. If we use fixed, arbitrary fidelity values $K$, one can always detect a range of $C$ values for which no MEMS characterized by $K$ exist. This feature constitutes a great difference with respect to what happens for the general mixed states case discussed above, where one has many different states compatible with a given value of the concurrence $C$. Such is the case for IH states, for which we can average such magnitudes as we did earlier for general mixed states in Sec. III. We must mind this difference between MEMS and IH states, if we want to compare MEM results to IH ones. As stated before, for an arbitrary
MEM state the concurrence, say $C=K$ is fixed, and so is the value of $F_{\min }$. But for the same $K$ value there exist many IH states characterized by a wide range of possible $F_{\min }$ values.

In order to be able to compare IH and MEM states we have used the following criterion: consider those IH states of concurrence $C=K$ with an $F_{\min }$ value greater than that pertaining to the associated $K$ MEM state. In Fig. 5 we depict $\tau / T_{L . B o u n d}$ vs $C$ for MEMS and for those IH states that fulfill the above criterion. The behavior of separable states, with exceedingly large $\tau / T_{L . B o u n d}$ values, is very different from that of highly entangled ones. The ensuing differences are larger than for the general mixed states studied in the preceding section. There is a clear difference between the two MEM zones arising out of the $x$-MEM parametrization. For weakly entangled states, $\tau / T_{L \text {.Bound }}$ achieves very high values. For highly entangled states (the second MEM zone), the situation is the opposite. In this last zone $\tau / T_{L \text {.Bound }}$ tends to 


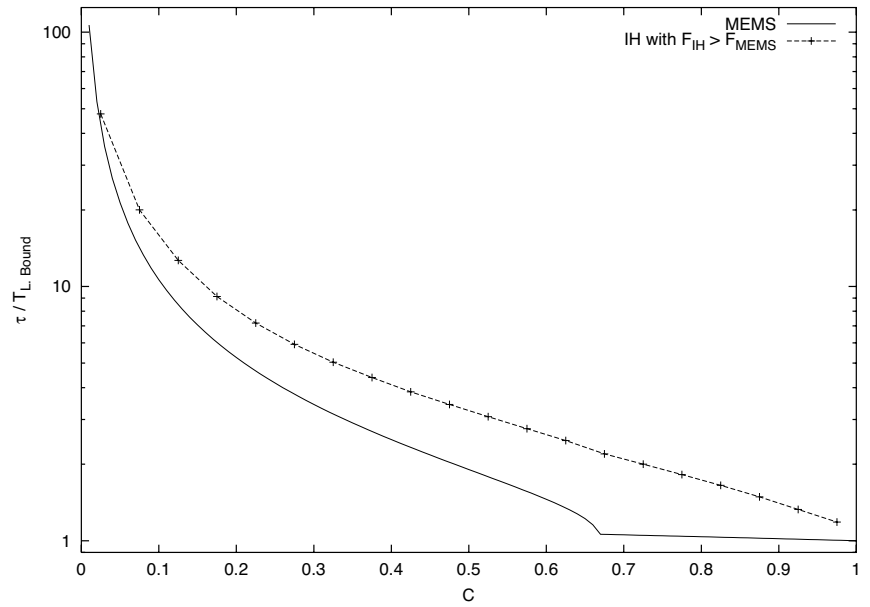

FIG. 5. $\tau / T_{L . \text { Bound }}$ for MEMS with Hamiltonian $H_{I I}$ and the corresponding average for the $\mathrm{IH}$ case. See text for details.

saturate its lower bound. The IH states have a similar behavior than the MEMS, although its corresponding average of the ratio $\tau / T_{L . \text { Bound }}$ is always greater than the corresponding MEMS ratio.

\section{CONCLUSIONS}

For mixed states of bipartite systems ruled by a general local Hamiltonian we have put forward rather convincing evidence of the clear correlation extant between concurrence and speed of time evolution. The more entangled an initial state $\rho_{1}$ is, the less time (in units of $T_{\text {L.Bound }}$ ) it takes to evolve to another state $\rho_{2}$, no matter what the $\rho_{1}-\rho_{2}$ fidelity is. In the case of pure states the correlation is strong for states that evolve to a minimum of the fidelity. For some special mixed states, namely, the so-called maximally entangled mixed states, the correlation between concurrence and the speed of time evolution becomes more acute than in the case of general mixed states.

\section{ACKNOWLEDGMENTS}

This work was partially supported by the MEC grant FIS2005-02796 (Spain) and FEDER (EU) and by CONICET (Argentine Agency). A. Borrás acknowledges support from the FPU grant AP-2004-2962 (MEC-Spain).
[1] V. Giovanetti, S. Lloyd, and L. Maccone, Europhys. Lett. 62, 615 (2003).

[2] V. Giovannetti, S. Lloyd, and L. Maccone, Phys. Rev. A 67, 052109 (2003).

[3] M. A. Nielsen and I. L. Chuang, Quantum Computation and Quantum Information (Cambridge University Press, Cambridge, 2000).

[4] Introduction to Quantum Computation and Information, edited by H.-K. Lo, S. Popescu, and T. Spiller (World Scientific, River Edge, 1998).

[5] The Physics of Quantum Information, edited by D. Bouwmeester, A. Ekert, and A. Zeilinger (Springer, Berlin, 2000).

[6] C. Tsallis, S. Lloyd, and M. Baranger, Phys. Rev. A 63, 042104 (2001).

[7] J. Batle, M. Casas, A. R. Plastino, and A. Plastino, Phys. Lett. A 296, 251 (2002).

[8] J. Batle, M. Casas, A. R. Plastino, and A. Plastino, Phys. Lett. A 298, 301 (2002).

[9] J. Batle, A. R. Plastino, M. Casas, and A. Plastino, Eur. Phys. J. B 35, 391 (2003).

[10] J. Batle, M. Casas, A. Plastino, and A. R. Plastino, Phys. Rev. A 71, 024301 (2005).

[11] J. Anandan and Y. Aharonov, Phys. Rev. Lett. 65, 1697 (1990).

[12] N. Margolus and L. B. Levitin, Physica D 120, 188 (1998).

[13] C. M. Caves and P. D. Drummond, Rev. Mod. Phys. 66, 481 (1994).

[14] S. Lloyd, Nature (London) 406, 1047 (2000).

[15] P. Kosiński and M. Zych, Phys. Rev. A 73, 024303 (2006).
[16] Shi-Biao Zheng and Guang-Can Guo, Phys. Rev. Lett. 85, 2392 (2000).

[17] Guo-Ping Guo, Chuan-Feng Li, Jian Li, and Guang-Can Guo, Phys. Rev. A 65, 042102 (2002).

[18] E. Solano, G. S. Agarwal, and H. Walther, Phys. Rev. Lett. 90, 027903 (2003)

[19] S. V. Prants, M. Yu. Uleysky, and V. Yu. Argonov, Phys. Rev. A 73, 023807 (2006).

[20] J. Batle, M. Casas, A. Plastino, and A. R. Plastino, Phys. Rev. A 72, 032337 (2005); 73, 049904(E) (2006).

[21] W. J. Munro, D. F. V. James, A. G. White, and P. G. Kwiat, Phys. Rev. A 64, 030302(R) (2001).

[22] S. Ishizaka and T. Hiroshima, Phys. Rev. A 62, 022310 (2000).

[23] N. A. Peters, J. B. Altepeter, D. A. Branning, E. R. Jeffrey, Tzu-Chieh Wei, and P. G. Kwiat, Phys. Rev. Lett. 92, 133601 (2004); 96, 159901(E) (2006).

[24] M. Barbieri, F. De Martini, G. Di Nepi, and P. Mataloni, Phys. Rev. Lett. 92, 177901 (2004).

[25] M. Ziman and V. Bužek, Phys. Rev. A 72, 052325 (2005).

[26] A. Aiello, G. Puentes, D. Voigt, and J. P. Woerdman, quant-ph/ 0603182.

[27] K. Zyczkowski, P. Horodecki, A. Sanpera, and M. Lewenstein, Phys. Rev. A 58, 883 (1998).

[28] K. Zyczkowski, Phys. Rev. A 60, 3496 (1999).

[29] M. Pozniak, K. Zyczkowski, and M. Kus, J. Phys. A 31, 1059 (1998).

[30] K. Bhattacharyya, J. Phys. A 16, 2993 (1983).

[31] P. Pfeifer, Phys. Rev. Lett. 70, 3365 (1993). 